\title{
A STUDY OF THE AGGLUTININ RESPONSE IN PATIENTS WITH MENINGOCOCCIC MENINGITIS ${ }^{1}$
}

\author{
BY HARRY F. DOWLING, RUTH L. MAYER, LEWIS K. SWEET, \\ AND EDITH DUMOFF-STANLEY
}

\author{
(From the George Washington Medical Division and the Pediatric Service (Dr. Sweet), Gallinger \\ Municipal Hospital, and the Departments of Medicine and Pediatrics, \\ George Washington University, Washington, D. C.)
}

(Received for publication July 12, 1944)

Although many workers have discussed the development of humoral antibodies in patients with meningococcic infections, the results of their observations have been irregular and at times conflicting. It has recently been concluded (1) that, of all the methods available at present, the agglutination test is the most reliable. These workers were unable to achieve high agglutinin titers by the use of the usual method of incubation for 2 hours at $37.5^{\circ} \mathrm{C}$., followed by 18 hours in the refrigerator, but with the use of the "thread test" they obtained consistent titers ranging up to $1: 128$ in patients with meningococcic meningitis and as high as 1:64 in contacts. We have likewise not observed sufficiently high titers with the orthodox method of incubation followed by refrigeration, and since the "thread test" is difficult to perform, except by persons highly trained in its use, we looked for another method which would be both reliable and practicable for routine use.

In the course of our search, we found that the use of centrifugation, in place of heating and refrigeration, gave consistently high titers on the sera of most patients during the course of meningococcic meningitis. The test is easy to perform and the results are highly specific. Sera obtained from patients with a variety of other diseases, including pneumococcic pneumonia and meningitis, gonococcic cervicitis, arthritis and meningitis and streptococcus viridans endocarditis, usually showed no agglutinins at all. In the occasional case where they did appear, the agglutinin titer was $1: 2,1: 4$, or, in one instance, 1:8. One patient with lymphocytic choriomeningitis exhibited an agglutinin titer of $1: 64$ on the fourth day of her illness and titers

1 This study was aided by a grant from the Lederle Laboratories, Inc. of $1: 32$ in all of the 5 other specimens of sera examined, the last one having been obtained on the fourteenth day of the disease. We are unable to account for these titers, which were so much higher than any others found in patients with infections not caused by the meningococcus, unless the patient might possibly have been a carrier of meningococci. This concept is strengthened by the fact that she was admitted during the peak of the meningitis epidemic, but cannot be proved, since no cultures were made from her throat.

The present report is a correlation between the results of our centrifugation-agglutination test and the course of the disease in patients with meningococcic infections.

\section{PROCEDURE}

Specimens of serum were collected during the course of the disease, usually at intervals of 1 or 2 days, from the day of admission until the day the patient was discharged from the hospital. In occasional patients, only 1 or 2 samples of serum were obtained.

Altogether 212 samples of serum were collected from 47 patients with typed meningococcic meningitis, and $\mathbf{7 0}$ specimens of serum from 20 patients who had meningitis resembling meningococcic meningitis clinically but from whose spinal fluid meningococci could not be cultured or typed by the immediate "quellung" method, although in some instances gram-negative diplococci were seen. A single agglutinin test was also done on each of 2 patients who appeared to have meningococcemia clinically, although the organisms were never cultured from the blood. All patients received sulfadiazine or sulfamerazine and 2 of them received penicillin.

The details of the technique of this test will be published elsewhere (2). Briefly, it was carried out as follows: Suspensions of strains of group I and group II alpha meningococci, freshly isolated from patients with the disease, were killed at $65^{\circ} \mathrm{C}$. and suspended in physiological salt solution without preservative. Serial dilutions of the patient's serum were prepared, and an equal amount of the diluted, killed suspension added to each tube, as in the usual agglutination test. Instead of incubation and 
TABLE I

Agglutinin titers achieved in cases of group I meningococcic meningitis

\begin{tabular}{|c|c|c|c|c|c|c|c|c|c|}
\hline \multirow[b]{2}{*}{ Titer } & \multicolumn{8}{|c|}{ Day of disease } & \multirow[b]{2}{*}{ All cases } \\
\hline & 1 to 5 & 6 to 10 & 11 to 15 & 16 to 20 & 21 to 25 & 26 to 30 & 30 and over & $\begin{array}{c}\text { Date of } \\
\text { onset not } \\
\text { known }\end{array}$ & \\
\hline $\begin{array}{l}0 . \\
1: 2 \\
1: 4 \\
1: 8 \\
1: 16 \\
1: 32 \\
1: 64 \\
1: 128 \\
1: 256 \\
1: 512\end{array}$ & $\begin{array}{r}37 \\
1 \\
3 \\
0 \\
4 \\
1 \\
7 \\
5 \\
2 \\
0\end{array}$ & $\begin{array}{r}8 \\
1 \\
3 \\
1 \\
1 \\
6 \\
4 \\
9 \\
11 \\
0\end{array}$ & $\begin{array}{r}3 \\
1 \\
1 \\
4 \\
1 \\
2 \\
2 \\
10 \\
11 \\
1\end{array}$ & $\begin{array}{l}6 \\
1 \\
4 \\
0 \\
0 \\
0 \\
4 \\
1 \\
6 \\
1\end{array}$ & $\begin{array}{l}3 \\
0 \\
0 \\
1 \\
0 \\
0 \\
1 \\
1 \\
1 \\
0\end{array}$ & $\begin{array}{l}3 \\
0 \\
0 \\
0 \\
0 \\
0 \\
0 \\
0 \\
1 \\
0\end{array}$ & $\begin{array}{l}9 \\
1 \\
0 \\
0 \\
0 \\
0 \\
0 \\
0 \\
0 \\
0\end{array}$ & $\begin{array}{l}2 \\
0 \\
0 \\
0 \\
0 \\
0 \\
1 \\
1 \\
0 \\
0\end{array}$ & $\begin{array}{r}71 \\
5 \\
11 \\
6 \\
6 \\
9 \\
19 \\
27 \\
32 \\
2\end{array}$ \\
\hline
\end{tabular}

refrigeration, however, the tubes were immediately centrifuged at 2,000 revolutions per minute for 20 minutes, after which the agglutinin titer could be read immediately.

\section{RESULTS}

Table I shows the agglutinin titers found on the 188 specimens of serum from the 44 patients with meningitis caused by group I meningococci to whom no antimeningococcic serum was given. All of the titers shown in the table were obtained with suspensions of group I meningococci, while a titer of zero was obtained with suspensions of group II alpha meningococci. In the case of 1 patient, not shown in the table, the meningococci found in the spinal fluid were typed as group I, while a single specimen of serum taken on the twentieth day of the disease was positive in a titer of 1:1024 for group II alpha and negative for group I.

It will be seen that during the first 5-day period after the onset of the meningitis the agglutinin titer was zero in the majority of instances. The titer for most patients rose considerably during the next 2 periods, . being 1:64 or above in the majority of cases from the sixth through the tenth day and 1:128 or above from the eleventh through the fifteenth day. Thereafter, the titer usually fell rather rapidly. In the period between the sixteenth and twentieth days, in about half of the cases, the titer was 1:4 or less, while in the other half, the titer was still high, 1:64 to $1: 512$. Except for 1 titer of $1: 256$ on the thirtieth day of the disease and 1 titer of $1: 2$ on the thirty-fifth day, all agglutinin titers had returned to zero after the twenty-fifth day, and in these 2 cases a specimen of serum taken still later gave an agglutinin titer of zero.

In Figure 1 the median agglutinin titers for each 5-day period are shown by solid circles, while the solid line represents the smoothed curve of the values which were obtained in all the patients with group I meningococcic meningitis in whom the day of the onset of the disease was known. The initial spinal fluid in all these patients was examined before treatment was begun, and the number of meningococci was counted in the average high-power field in a stained smear of the sediment after centrifugation. If there were an average of 5 or more organisms per high-power field, these were

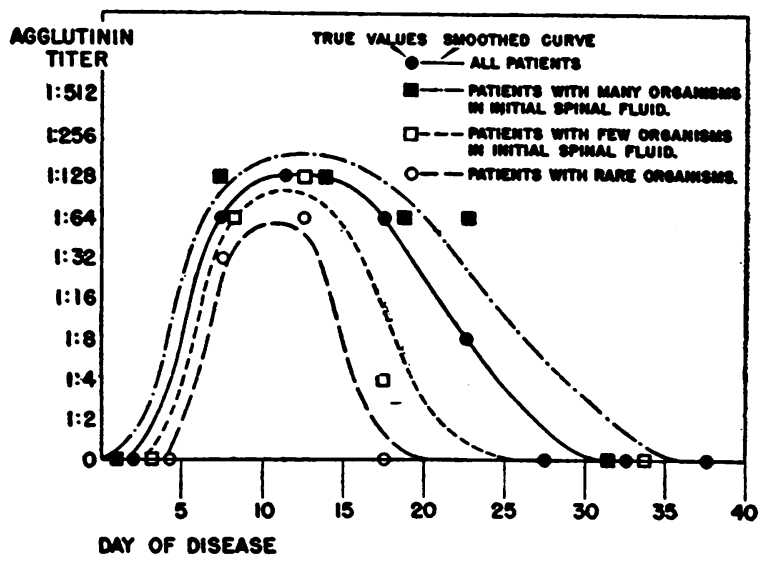

Fig. 1. Median Agglutinin Titers in Patients with Group I Meningococcic Meningitis, Arranged by 5-day Periods from Date of Onset 
designated as "many" organisms. When 1 to 4 diplococci were present, they were considered to be "few" in number, and if less than 1 diplococcus per high-power field was seen, organisms were said to be "rare." From the figure it will be seen that, when "many" organisms were present in the initial spinal fluid, the agglutinin titers in the patient's serum usually rose to higher levels and fell more slowly than when only. a "few" organisms were present. When organisms were "rare" in the initial specimen, the maximal titer achieved was usually lower than in any of the other groups and the titer fell to zero sooner.

We have not been able to correlate the agglutinin titers achieved with any other factor with which we have compared them, such as, the age of the patient, the presence or absence of a positive blood culture, the presence of complications of the disease, the day of the disease on which specific treatment was begun, or the rapidity of response to specific treatment. Agglutinin titers were determined on 4 patients who subsequently died and who had group I meningococci in the spinal fluid. One of these had an agglutinin titer of $1: 32$ on the sixth day of the disease and died on the eighth day. The second had a titer of 1:64 on the fifth day and died on the seventh day. The third came in in coma so that the date of onset of his illness could not be ascertained. He had an agglutinin titer of $1: 128$ on the third day of his hospital stay and died on the following day. The fourth patient was also unable to give a history. He had no agglutinins on the second and third days of hospitalization and died on the third day. Apparently the height of the agglutinin titer is not related to the outcome of the disease, at least in patients receiving chemotherapy.

One patient, not included in Table I, had no agglutinins on the fourth day of his disease. Because he was seriously ill, he was given $80 \mathrm{ml}$. of polyvalent antimeningococcic serum the same day. One hour later he had a titer of 1:64 for group I meningococci and none for II alpha. Eleven days later the titer for group I was 1:128 and thereafter it gradually descended to $1: 8$ on the fifty-third day of the disease, when he was discharged.
Specimens of serum for agglutination were obtained on 1 patient whose spinal fluid was found to contain group II alpha meningococci. He was admitted on the fourth day of the disease. Thirteen specimens of serum were obtained. Agglutinin titers with a suspension of group II alpha meningococci started at zero on the fourth day of his illness, slowly rose to 1:64 on the eleventh day, and fell to a titer of $1: 4$ on the nineteenth day when he was discharged from the hospital completely recovered. All agglutinin controls for group I meningococci were negative.

It is of interest that among the 20 patients on whom 5 or more specimens of serum were tested, all had some agglutinins at one time or another. In 1 patient, the highest titer was $1: 2$; in another, it was $1: 4$; in 2 more, $1: 8$; while in all the others, the highest titer was $1: 32$ or more.

TABLE II

\begin{tabular}{|c|c|c|c|}
\hline \multirow{2}{*}{$\underset{\text { titer }}{\text { Agglutinin }}$} & \multicolumn{2}{|c|}{$\begin{array}{l}\text { Tests on patients with } \\
\text { meningitis }\end{array}$} & \multirow{2}{*}{$\begin{array}{l}\text { Tests on pa- } \\
\text { tients with } \\
\text { apparent } \\
\text { meningo- } \\
\text { coccemia }\end{array}$} \\
\hline & $\begin{array}{c}\text { Organisms } \\
\text { seen on smear }\end{array}$ & $\begin{array}{c}\text { No organisms } \\
\text { seen }\end{array}$ & \\
\hline $\begin{array}{l}0 \\
1: 2 \\
1: 4 \\
1: 8 \\
1: 16 \\
1: 32 \\
1: 64 \\
1: 128 \\
1: 256 \\
1: 512\end{array}$ & $\begin{array}{c}23^{*} \\
3^{*} \\
2 \\
2 \\
0 \\
1 \\
0 \\
1 \\
1 \\
1\end{array}$ & $\begin{array}{r}19 \\
3 \\
1 \\
0 \\
2 \\
3 \\
1 \\
3 \\
0 \\
2\end{array}$ & $\begin{array}{l}0 \\
0 \\
1 \\
0 \\
0 \\
0 \\
0 \\
1 \\
0 \\
0\end{array}$ \\
\hline $\begin{array}{l}\text { Total number of } \\
\text { tests }\end{array}$ & 34 & 34 & 2 \\
\hline
\end{tabular}

* Agglutinin titers all for group I (and zero for group IIa) except in the case of one patient where they were positive for group II and zero for group I.

In Table II are shown the agglutinin titers in patients who had conditions clinically resembling meningococcic meningitis, or meningococcemia, although no organisms were cultured or typed from the spinal fluid or blood. In the second column are shown the tests done on patients with meningitis in whose spinal fluid gramnegative diplococci were seen, and in the third column, those done on patients whose spinal 
fluid revealed no organisms. In the fourth column are 2 tests done on the serum of patients with a disease clinically resembling meningococcemia whose blood cultures were negative. It will be seen that agglutinin titers at all levels up to 1:512 were obtained in these cases, and that there was no significant difference between the titers, whether organisms were seen in the spinal fluid or not. When a comparison is made with Table $I$, it is seen that there was a tendency for higher agglutinin titers to occur in the sera of patients from whom meningococci were cultured. Although the number of cases was not great enough to permit a valid comparison, we found that the titers in the patients who showed no organisms on smear and culture corresponded closely with the titers in the patients with rare or no organisms on smear but with positive cultures.

\section{DISCUSSION}

Because there has been no complete study of the rise, duration, and fall of agglutinin titers during the course of meningococcic meningitis, we have undertaken such a study by testing sera from 67 patients with this disease and 2 patients with an infection clinically resembling meningococcemia. Group I or group II alpha meningococci were typed from the spinal fluid of 47 of these patients. Organisms morphologically resembling meningococci were seen in the spinal fluid of 10 additional patients, while in 10 patients, the disease was diagnosed on clinical grounds alone. The agglutinins were specific for the group of meningococcus typed from the spinal fluid, except in 1 instance in which group I meningococci were found, while a single specimen of serum on the twentieth day of the disease showed an agglutinin titer of 1:1024 for group II alpha and none for group I. We are unable to account for this discrepancy. Since the agglutination test was done several weeks after the patient was discharged from the hospital, we could not repeat the typing or secure other samples for agglutination. The agglutination test was clear-cut in its results, so that, if a mistake was made, it may have been in the typing at the time of admission or in recording the results of the typing.

When we considered only the patients with typed meningococcic meningitis in whom the day of onset was known, we found that the median agglutinin titer rose from zero in the first 5-day period after the onset of the meningitis to 1:64 and 1:128 in the second and third 5-day periods, respectively. Thereafter the median titer fell until it reached zero between the twentyfifth and thirtieth day, although in individual cases the titers remained elevated until as late as the fifty-third day of the disease. If we were to add the titers for the untyped cases to those of the typed group, the median values for all cases would remain the same.

In a recent study (3) of agglutinins in patients with meningococcic meningitis, the first significant titers (1:30 or more by their technique) were found to occur on the seventh day of the disease and the last ones on the forty-ninth day. Titers went as high as 1:240 during the peak period, which was stated to be around the twentyfirst day. Our results were similar to these except that the titers we obtained were higher in general, presumably due 'to the fact that the previous investigators used the usual heatfollowed-by-refrigeration method. In the few cases which Thomas and his associates (1) report, the trend of agglutinin titers is also comparable with those that we have encountered.

In general, agglutinins were found to rise to higher levels the greater the number of meningococci found in the initial specimen of spinal fluid, and to be lowest when only a rare organism was present at the time of the first spinal puncture. It is interesting in this connection that the chance of recovery has been shown (4) to be inversely proportional to the number of organisms in this initial specimen of spinal fluid. Since only 4 patients in the present group died, the agglutinin titer could not be related to prognosis, although it was observed that 3 of these patients had agglutinins in significantly high titers before death. The agglutinin response could not be correlated with any other factors that we were able to study.

In view of the fact that most of our patients with meningitis showed a high titer of agglutinins at some time during the course of the disease, if a sufficient number of specimens were examined, while, on the other hand, only 1 of the patients with other infectious diseases showed a 
titer above $1: 8$, this test is obviously useful in detecting the presence of meningococcic infections when organisms cannot be typed from the blood or spinal fluid. Certain investigators (5) have diagnosed meningococcemia from the clinical features of their cases plus the presence of agglutinins as demonstrated by the "thread test." Since we have obtained a significantly high titer in one case of clinical meningococcemia and a suggestive titer in another, we would recommend that the centrifuge-agglutination test be employed when meningococcemia is suspected. From present evidence, titers above 1:64 or rising titers would confirm the clinical diagnosis of a meningococcic infection.

\section{SUMMARY AND CONCLUSIONS}

1. Two hundred and eighty-four specimens of serum from 69 patients with meningococcic meningitis or meningococcemia were examined for agglutinins by means of a test using centrifugation instead of the usual method of heating at $37.5^{\circ} \mathrm{C}$. followed by refrigeration.

2. Agglutinin titers were specific for the group of meningococcus isolated from the spinal fluid of the patient except in one instance. The titers ranged from zero to $1: 1024$. The median titer in typed group I patients rose to
$1: 128$ for the period from the eleventh to the fifteenth day, inclusive, and fell to zero in the period between the twenty-fifth and thirtieth days.

3. The median agglutinin titer rose to higher levels the greater the number of organisms present in the initial specimen of spinal fluid.

4. The centrifugation-agglutination test is a simple, rapid, and efficient method which may be used to diagnose cases of meningococcic infections where organisms cannot be cultured from the body fluids.

\section{BIBLIOGRAPHY}

1. Thomas, L., Smith, H. W., and Dingle, J. H., Investigations of meningococcal infection. II. Immunological aspects. J. Clin. Invest., 1943, $22,361$.

2. Mayer, R. L., and Dowling, H. F., To be published.

3. Van Rooyen, C. E., and Morris, J. C., Bacteriological researches on cases of cerebrospinal meningitis, convalescents and carriers. J. Roy. Army M. Corps, 1941, 76, 200.

4. Lepper, M. H., Sweet, L. K., and Dowling, H. F., The treatment of meningococcic infections with sulfadiazine and sulfamerazine (sulfamethyldiazine, monomethylsulfadiazine). J.A.M.A., 1943, 123, 134.

5. Daniels, W. B., Solomon, S., and Jaquette, W. A., Jr., Meningococcic infection in soldiers. J.A.M.A., $1943,123,1$. 